\section{ARS Tech}

Authors retain copyright

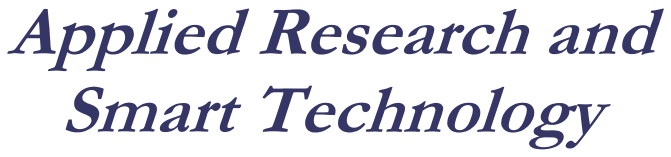

Journal homepage: www.journals2.ums.ac.id/index.php/arstech

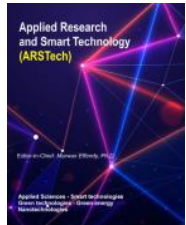

ISSN 2722-9645

Research Article

\title{
A simplified approach for single carrier visible light communication transceiver using off-the-shelf components
}

\author{
Augustus E. Ibhaze ${ }^{1,{ }^{*}}$, Patience E. Orukpe ${ }^{2}$, Frederick O. Edeko ${ }^{3}$ \\ ${ }^{1}$ Department of Electrical and Electronics Engineering, University of Lagos, Akoka, Yaba, Lagos, 100213, Nigeria. \\ ${ }^{2,3}$ Department of Electrical and Electronic Engineering, University of Benin, Benin City, 300283, Nigeria. \\ ${ }^{*}$ Corresponding author: eibhaze@unilag.edu.ng
}

Permalink (DOI): $\underline{\text { https://doi.org/10.23917/arstech.v1i2.102 }}$

\begin{tabular}{|c|c|}
\hline ARTICLE INFO & ABSTRACT \\
\hline Article history: & Over the past decades, the demand for high-speed data transmission has grown \\
\hline Received 09 Jul 2020 & exponentially, necessitating broadband technology integration to satisfy the \\
\hline Kecerved 09 Jul 2020 & emerging data-hungry society. This concern has engendered the proposition of a \\
\hline Revised 20 Aug 2020 & simplified optical wireless transceiver module using off-the-shelf electronic \\
\hline Accepted 03 Oct 2020 & devices. According to the transistor to transistor logic levels, the transmitter \\
\hline Available online 30 Nov 2020 & module was made up of a single negative-positive-negative (NPN) transistor \\
\hline Published regularly 15 Dec 2020 & (LED). In contrast, the receiver was made up of a photodetector (solar) module \\
\hline Keywords: & for photons detection and back-conversion to electrical signals. The received \\
\hline Light-emitting diode & signal was passed through a four-stage amplification process to remove the \\
\hline Photodetector & ambient noise and retrieve the data signal. The last transceiver module was \\
\hline Single carrier & demonstrated using personal computers. It was observed to perform at different \\
\hline Transistor & optimum points consistent with exposure to fluorescent light, daytime, and \\
\hline Visible light communication & $\begin{array}{l}\text { night-time operations consequent upon the induced ambient light interference } \\
\text { at data rates constrained by the communication device interface. }\end{array}$ \\
\hline
\end{tabular}

\section{INTRODUCTION}

Wireless communication has gradually evolved over the years and has become the mainstay for global convergence relative to increasing broadband infrastructure demands. Visible light is a promising band in the electromagnetic spectrum, which occupies the terahertz frequency range with adequate bandwidth and capabilities for broadband integration [1-3]. Compared with its radio frequency counterpart, which has been well exploited for communication purposes due to its wide-area coverage, capacity, and evolved interference mitigation techniques [4] [5], the visible light spectrum has been under rigorous investigation. However, its potential has never been fully exploited due to conventional light sources' inability to handle rapid modulation [6]. The advent of the Light Emitting Diode (LED), particularly the first white LED, opened up an entirely new range of possibilities in Visible 
Light Communication (VLC) and brought it to the forefront of research. The VLC system [Z] has resulted in throughputs up to gigabits per second in offline networks [ $\underline{8}-10]$ due to the adoption of high-end digital signal conditioning techniques in the domain of multicarrier schemes. Since the research community is yet to record a significant breakthrough in hardware support for such methods within the wireless optical field in online applications, single-carrier systems are being investigated for capacity enhancement within small cells [11]. The most basic process of sending and receiving data signals in VLC involves modulation, driving the light source, detection, and demodulation to retrieve the transmitted data.

In a study, a pulse width modulation system has been successfully investigated [12]. The single carrier (SC) system was specifically designed to transfer texts between two Personal Computers (PCs) and achieve up to $1.5 \mathrm{kbps}$. As a point to point, the system was in view, an increase in the receiver's angle reduced the modulation accuracy. The transmitter side had an analogue front end consisting of high-power white phosphorus LED capable of limiting the sufficient bandwidth due to the yellow phosphor's slow response. An improvement in throughput was recorded in [13], using QAM (quadrature Amplitude Modulation), a variant of the PAM (Pulse Amplitude Modulation). The model adopted high-power white phosphorescent LED module (OSTAR LE CW E2B) in the transmitter front end, which is critical when considering energy-efficient modules. A signal amplification-based transceiver for optical wireless communication was proposed [14]. The design utilized a laser diode and an amplification circuit for signal transmission while photodiode and an amplification module for signal reception. Although the authors were able to achieve device-to-device communication using VLC techniques, it was reported that the achievable data rate could be limited due to the interfaces and therefore proposed the design of propriety VLC-based interfaces. Other attempts in optical single carrier implementations have been well validated in [15][23] with a trade-off between the transceiver design complexity and performance metrics. Attempts in minimizing the design complexity by implementing a simplified design using off-the-shelf electronic devices have engendered the model presented in this work.

\section{RESEARCH METHODOLOGY}

The experimental setup is as shown in Figure 1, detailing the wireless optical transceiver module's implementation. The data supplied from the PC was fed into the USB to serial converter, which acts as an interface between the source of the PC data and the circuit and converts the data from the $\mathrm{PC}$ into a signal that could be modulated for transmission. The modulation method was achieved by intensity modulation (IM) via the LED switching using transistor Q1 shown in Figure 2. This switching method gave rise to the on-off keying (OOK) technique of the LED transmitter front end, as depicted in the LED driver module shown in Figure 2.

The photodiode directly detected the emitted photons and produced currents corresponding to the transmitted signal. This signal was then amplified, shaped, and demodulated for data recovery.

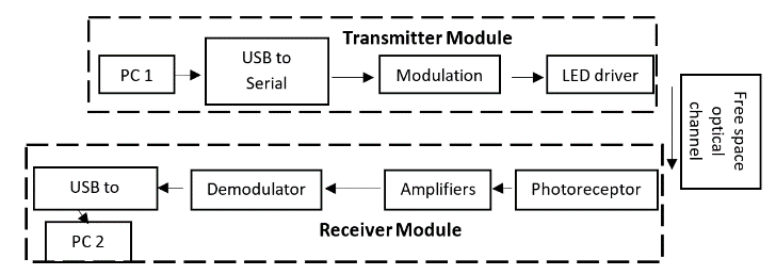

Figure 1. Transceiver Block diagram.

A significant limitation in implementing VLC systems was ambient noise within the system channel capable of degrading the received signal. For the application to be functional, there must be a way to filter the effect of noise. The adaptive minimum voltage removal technique proposed in [23][24] was implemented. A proteus simulation was run at every stage to ensure the validity of the circuit subsections. The encoder was the Max232 IC, which converts the USB (Universal Serial Bus) to Serial (RS232) input to TTL (TransistorTransistor Logic) signal levels. The TTL output was a unipolar non-return to zero (NRZ) line coding format, which encodes a positive voltage as bit 1 and zero voltage as bit 0 . In a similar tone, the parity in the serial transmission protocol could allow for single error detection. Therefore, it was expected that a more sophisticated error detection and correction coding scheme could be implemented to improve the overall performance of this proposed design in future work. The USB to Serial converter and the Max232 was not included in the circuit simulations. A mock circuit was simulated and tested with serial data generated by the virtual terminal on proteus shown in Figure 2.

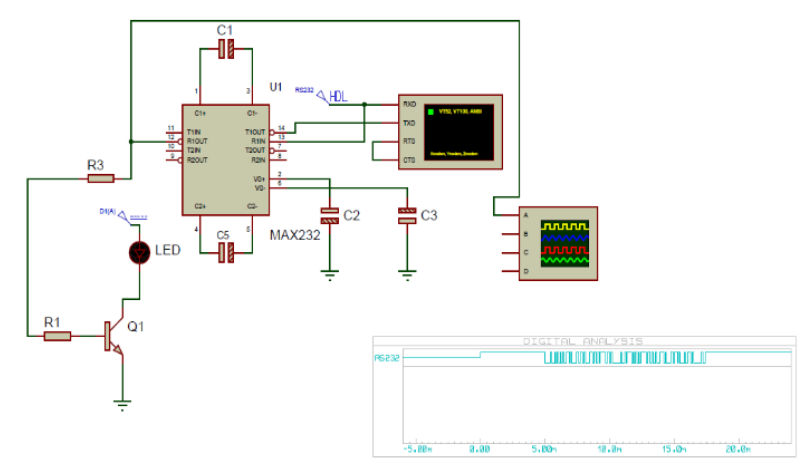

Figure 2. Transmitter circuit. 
The major component of the LED driving circuit is the transistor, as seen in Figure 2. The pulse signal from the MAX232 IC turns the transistor on and off according to voltage levels. The received signal then undergoes a four-stage process to retrieve the data after photodetection. The amplification block is divided into four sections, shown in Figure 3, while the receiver module's circuit realization is displayed in Figure 4.

\begin{tabular}{|c|c|c|c|}
$\begin{array}{c}\text { Minimum } \\
\text { amplifier }\end{array}$ \\
$\begin{array}{c}\text { Voltage level } \\
\text { detector }\end{array}$
\end{tabular}$\longrightarrow \begin{gathered}\text { Differential } \\
\text { Amplifier }\end{gathered} \longrightarrow \begin{gathered}\text { Non- } \\
\text { inverting }\end{gathered}$

Figure 3. Amplification block.

The voltage output characteristic of the photoreceptor was used due to ease of measurement. The op-amp circuit configuration produced an amplified output signal of the voltage input obtained from the photodetector.

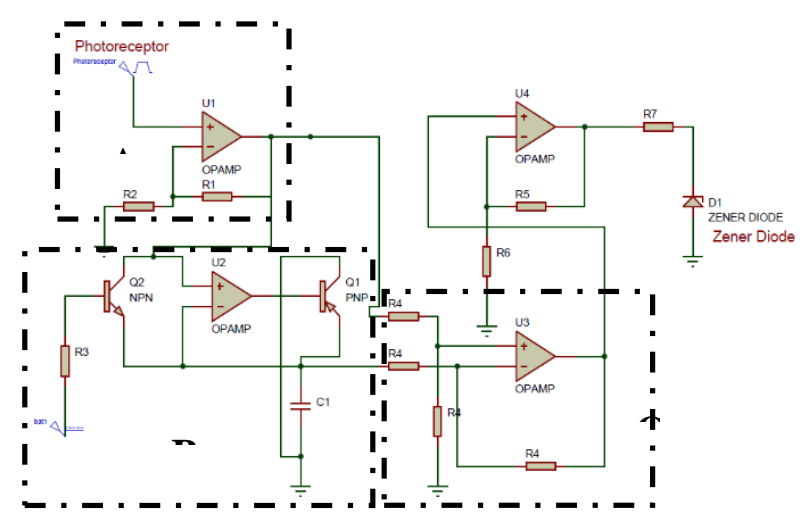

Figure 4. Receiver circuit.

The part labelled A in Figure 4 is the non-inverting amplifier stage of the receiver circuit. The node between the feedback resistor RF and R1 has a voltage indicated by $V_{x}$ :

$$
\begin{aligned}
& V^{-}=V_{x} \\
& V_{x}=\frac{R 1}{R 1+R f} \times V_{\text {out }}
\end{aligned}
$$

In a negative feedback configuration,

$$
V^{+}=V^{-} \text {(due to a virtual short) }
$$

Therefore,

$$
V^{+}=V_{\text {in }}
$$

and also,

$$
V^{-}=V_{\text {in }}
$$

By combining (1) and (5), we obtain:

$$
V_{x}=V_{\text {in }}
$$

Hence, (2) becomes:

$$
V_{\text {in }}=\frac{R 1}{R 1+R f} \times V_{\text {out }}
$$

Therefore, the gain becomes:

$$
\frac{V_{\text {out }}}{V_{\text {in }}}=\frac{R 1+R f}{R 1}=1+\frac{R f}{R 1}
$$

For the minimum voltage detector shown in the labelled part B of Figure 4 to be functional, the capacitor C1 is essential. It acts as a memory element to store the minimum voltage. The voltage measured across the capacitor is the same as the voltage measured across the operational amplifier's inverting terminal. In the design, an NPN transistor is connected between the non-inverting terminal and inverting terminal of the operational amplifier. The capacitor's charging rate depends on the capacitance of C1 and emitter currents of both Q1 and Q2. If the amplitude of the voltage at the non-inverting terminal is below the voltage level at the inverting terminal, Q1's emitter current must be of greater magnitude than Q2's to ensure that the capacitor discharges instantaneously; therefore, the current-limiting resistor connected to Q2 must have high resistance. If the non-inverting voltage is greater than the inverting voltage of the operational amplifier, Q1 is switched off, and Q2's emitter current must maintain a threshold value such that the voltage across $\mathrm{C} 1$ does not exceed or drop below a onebit duration of the input signal voltage. The value of the battery $V_{S}$ and capacitor $\mathrm{C} 1$ had experimented within the proteus design environment before the optimum values were selected. The differential amplifier stage shown in part $\mathrm{C}$ of Figure 4 can be used to find the difference between the two voltages. As used here, it subtracts the offset voltage, the minimum voltage stored by capacitor C1. Assigning $V_{1}$ to be the minuend and $V_{2}$ to be the subtrahend, the output voltage $V_{o}$ can be expressed as a relationship between the values of the resistors.

$$
\frac{V 1-V^{-}}{R 1}=I_{1}, \frac{V 2-V^{+}}{R 3}=I_{2}, \frac{V^{-}-V o}{R 4}=I_{f}
$$

At the Summing Points, Eqn. (3) holds.

And by voltage division,

$$
V^{+}=\frac{R 3}{R 3+R 2} \times V 2
$$

If $V_{2}$ is zero, then,

$$
V_{0(-)}=-V 1 \frac{R 4}{R 1}
$$

If $V_{1}$ is zero, then,

$$
V_{0(+)}=V 2 \frac{R 3}{R 3+R 2} \times \frac{R 4+R 1}{R 1}
$$

Therefore,

$$
\begin{aligned}
& V_{0}=-V_{0(-)}+V_{0(+)} \\
& V_{0}=-V 1 \frac{R 4}{R 1}+V 2 \frac{R 3}{R 3+R 2} \times \frac{R 4+R 1}{R 1}
\end{aligned}
$$


If

$$
R 1=R 2=R 3=R 4
$$

Then,

$$
V_{0}=V 2-V 1
$$

The optical wireless receiver module for single carrier modulation, combining the specific modules for the reception process, is shown in Figure 5.

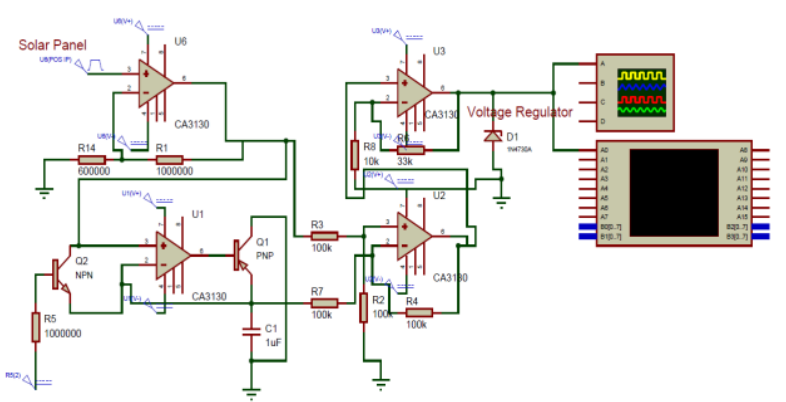

Figure 5: Receiver Test circuit.

\section{RESULTS AND DISCUSSION}

As the circuitry has no drivers with which the computer could interact, it was necessary to find an interface between the PC and the circuit. The most straightforward approach was to use a USB to Serial (TTL) converter. A USB to TTL converter is a device which converts information signal from the USB port to signals that can be processed by transistor base devices and other devices. The line of devices considered for implementing this research was the FTDI (Future Technology Devices International) chipset. The model used was the FT232r device. An NPN transistor was used as the control switch to modulate the transmitting LED. The base resistor required to push the transistor into saturation mode with the $5 \mathrm{~W}$ LED was estimated by,

$$
\text { power }=\frac{V^{2}}{R}
$$

where $\mathrm{V}=$ voltage requirement

and $\mathrm{R}$ is the inherent Resistance of the LED

The calculated resistance was $28.8 \mathrm{ohms}$

The LED's inherent resistance was determined using the power - resistance relation, with power and voltage values of $5 \mathrm{~W}$ and $12 \mathrm{~V}$, respectively. The base resistor required to enable an NPN transistor pulse driver was calculated.

where $V_{B E}$ is the Base Emitter Voltage

$V_{C C}$ is the power supply voltage

$I_{C}$ is the collector current

$I_{b}$ is the base current

$$
\begin{aligned}
& I_{c}=\frac{V_{c c}}{R_{c}} \text { when } V_{c e}=0 \\
& I_{b}=I c / \beta
\end{aligned}
$$

where $\beta$ is the current gain which is 100 for the 2N2222A model;

$$
\text { Since } I_{c}=0.41667 A, I_{b}=0.0041667 A
$$

For this investigation, the transistor was required to be in the ON-state when the bit level is high $(5 \mathrm{~V})$, therefore,

$$
\begin{aligned}
& \text { when } V_{i}=5 \mathrm{~V} \\
& \text { For silicon transistors }
\end{aligned}
$$

$0.0041667=(5 v-0.7) / \max$ base resistance

For the transistor to be $\mathrm{ON}$, the maximum base resistance required for the transistor to be in a high state is $1032 \mathrm{ohms}$. The experimental validation used to verify the circuit's logic was bread-boarded, as shown in Figure 6. The link parameters are shown in Table 1.

Table 1. System Parameters for the Transceiver VLC Link.

\begin{tabular}{lll}
\hline & Parameters & Values \\
\hline Source & Location of LED & $(0,0,0)$ \\
& Transmitted power & $<5 \mathrm{~W}$ \\
Receiver & Distance & $<15 \mathrm{~cm}$ \\
& Active Area & $6 \mathrm{~cm}^{2}$ \\
& Half-angle FOV & 60 \\
\hline
\end{tabular}

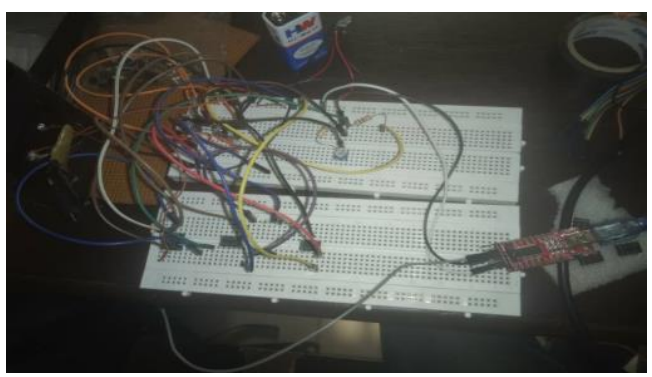

Figure 6. Transceiver testing.

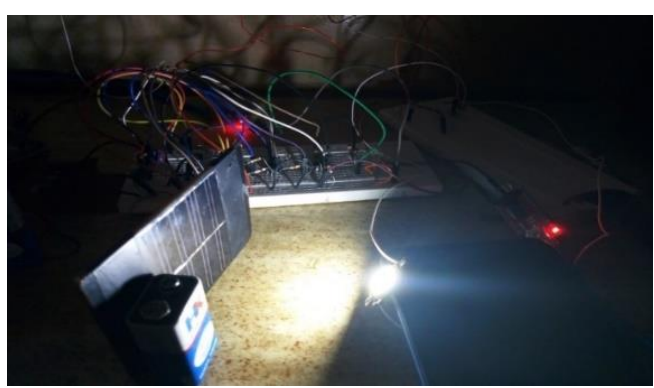

Figure 7. Transceiver Setup. 
Figure 7 to Figure 8 shows the single carrier optical transceiver module's outcome and its implementation for data communication between two Personal Computers (PCs) on a point-to-point link.

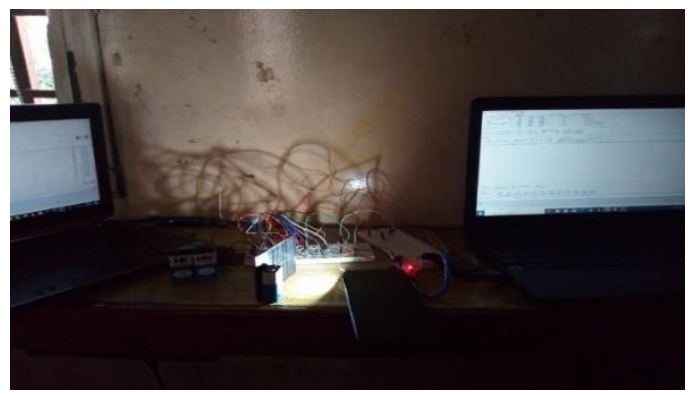

Figure 8. PC to PC Data Transaction.

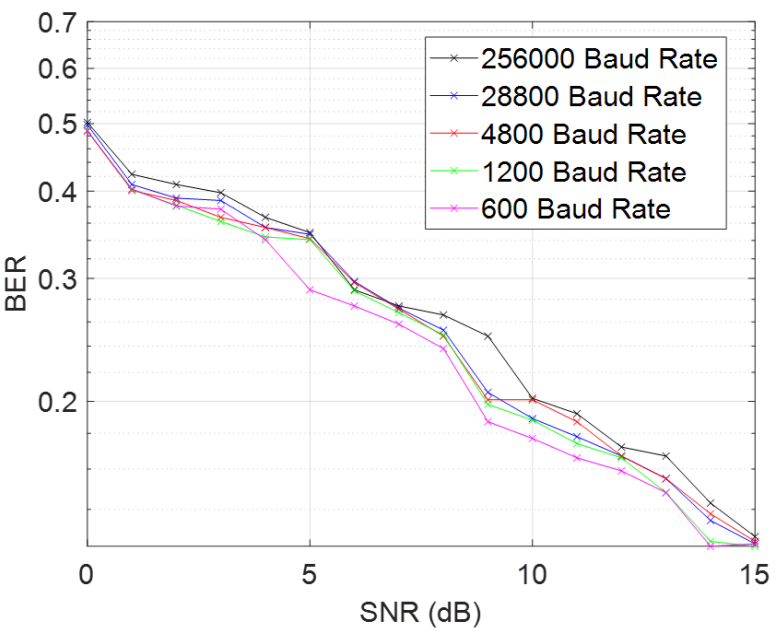

Figure 9. Error rate response during night time.

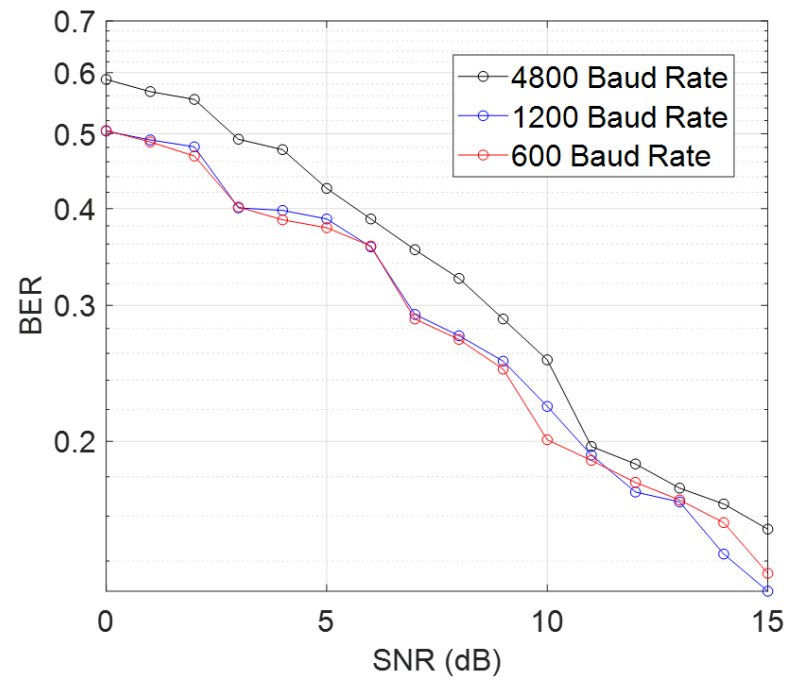

Figure 10. Error rate response during day time.

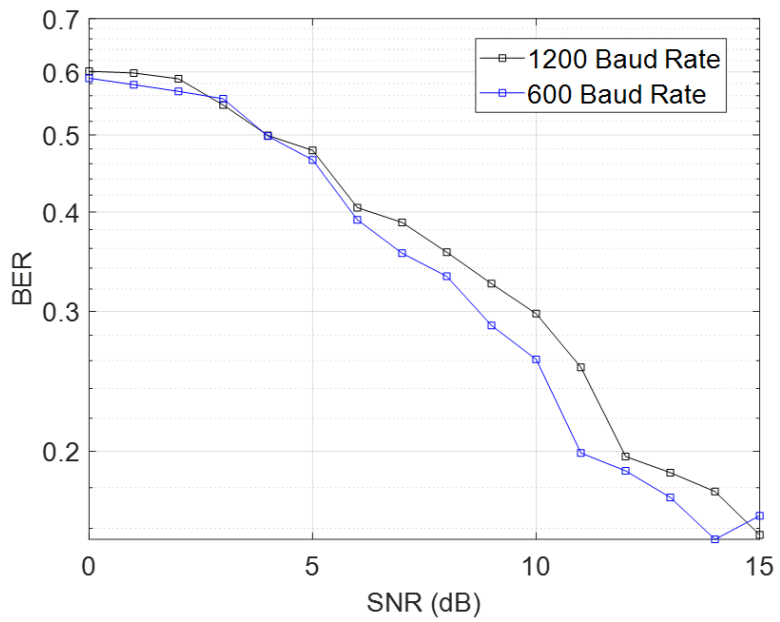

Figure 11. Error rate response under fluorescent light environment.

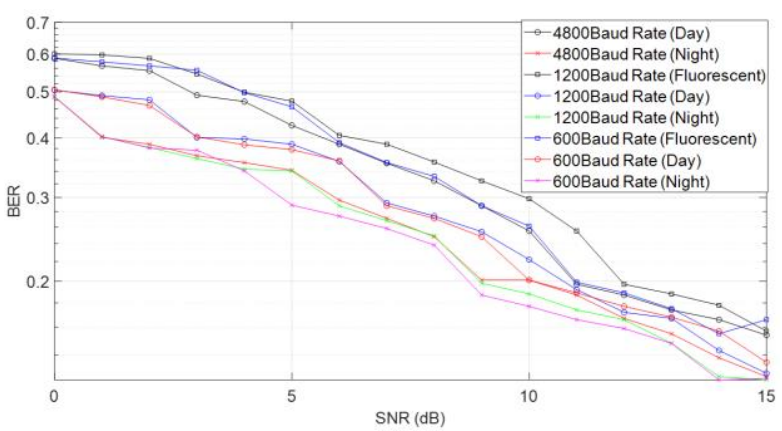

Figure 12. Comparison of Error Rate Response under different Ambient Light Conditions

Figure 9 to Figure 12 depicts the error rate response at varying baud rates under diverse ambient light conditions. Under ambient night-time light, the error-free transmission was achieved up to 256,000 baud rates as summarized in Table 3. The bit error rate (BER) response relative to increasing signal to noise ratio (SNR) is shown in Figures 9 to 12, respectively. The BER response is closely related at diverse baud rates as the transmitted signals were received undistorted due to the absence of ambient interfering sources at night-time. The effects of ambient daylight and florescent light were seen to have degraded the effective transmission rate considerably relative to high BER at lower SNR values. The BER response improves at higher $\mathrm{SNR}$ up to $15 \mathrm{~dB}$, as depicted in Figures 10 to 12 . Tables 2 and 4 provide a summary of the system performance under interfering light sources. Generally, the BER response is seen to deteriorate at higher baud rates irrespective of the operation mode for the apparent reason of having more bits constrained within the single wideband channel. Hence, with a proper ambient noise shielding effect, the transmission process can be enhanced for improved transmission performance irrespective of the days' time. 
Table 2. Effect of Ambient light on baud rate during the daytime.

\begin{tabular}{lll}
\hline Baud Rate & Text sent & Remark \\
\hline 600 & This is a test & The text was received successfully \\
1200 & This is a test & $\begin{array}{l}\text { The text was received successfully } \\
\text { There were errors in the received } \\
\text { text }\end{array}$ \\
\hline
\end{tabular}

Table 3: Effect of Ambient light on baud rate during night-time.

\begin{tabular}{|c|c|c|}
\hline Baud Rate & Text sent & Remark \\
\hline 600 & This is a test & The text was received successfully \\
\hline 1200 & This is a test & The text was received successfully \\
\hline 4800 & This is a test & $\begin{array}{l}\text { There were errors in the received } \\
\text { text }\end{array}$ \\
\hline
\end{tabular}

Table 4: Effect of Ambient light on baud rate under fluorescent light.

\begin{tabular}{lll}
\hline Baud Rate & Text sent & Remark \\
\hline 600 & This is a test & $\begin{array}{l}\text { While the tests were received } \\
\text { successfully, there was always noise } \\
\text { being computed during idle times. }\end{array}$ \\
1200 & This is a test & $\begin{array}{l}\text { While the tests were received } \\
\text { successfully, there was always noise } \\
\text { being computed during idle times. }\end{array}$ \\
\hline
\end{tabular}

\section{CONCLUSION}

This research has presented a simplified circuit model for visible light communication by implementing intensity modulation/direct detection (IM/DD) using an off-the-shelf LED and a photodetector. The error-free transmission is achieved during the night-time season at an average BER of 0.125 at $15 \mathrm{~dB}$ SNR at diverse baud rates due to the absence of interfering light sources. Under fluorescent light exposure and daytime operation, the system performance is seen to have degraded with an increase in the average BER response of 0.162 and 0.139 at $15 \mathrm{~dB}$, respectively. The transceiver module could be adapted for communication in application areas where external ambient light sources could be avoided, such as wireless sensor communication in tunnels, mines, and downhole exploration in the oil and gas field.

\section{CONFLICTS OF INTEREST}

The authors declare no conflicts of interest.

\section{ACKNOWLEDGEMENT}

The authors would like to thank the University of Lagos for supporting this research.

\section{REFERENCES}

[1] D. Karunatilaka, F. Zafar, V. Kalavally and R. Parthiban, "LED-based indoor visible light communications: State of the art", IEEE Communications Surveys \& Tutorials, vol. 17, no. 3, pp.1649-1678, thirdquarter 2015. https://doi.org/10.1109/COMST.2015.2417576.

[2] A.E. Ibhaze, P.E. Orukpe, and F.O. Edeko, "Li-Fi prospect in internet of things network", Advances in Information and Communication. FICC 2020, Advances in Intelligent Systems and Computing, vol. 1129, pp.272-280, 2020. https://doi.org/10.1007/978-3-030-39445-5_21.

[3] A.E Ibhaze, P.E Orukpe, and F.O Edeko, "High capacity data rate system: Review of visible light communications technology", Journal of Electronic Science and Technology, vol, 18, no. 3, 100055, 2020. https://doi.org/10.1016/j.jnlest.2020.100055.

[4] J.O. Abolade, A.E. Ibhaze, A.L. Imoize, and A.A. Atayero, "Cross layer enhanced adaptive handoff initiation technique", World Scientific News, vol. 120, no. 2, pp.154-167, 2019.

[5] C. Çeken, Y. Serhan, and A. Huseyin, "Interference aware vertical handoff decision algorithm for qos support in wireless heterogeneous networks", Computer Networks, vol. 54, no. 5, pp. 726-740, 2010. https://doi.org/10.1016/j.comnet.2009.09.018.

[6] L.U. Khan, "Visible light communication: Applications, architecture, standardization and research challenges", Digital Communications and Networks, vol. 3, no. 2, pp.78-88, 2017. https://doi.org/10.1016/j.dcan.2016.07.004.

[7] C. Han, X. Sun, and S. Cui, "Design of $100 \mathrm{Mbps}$ white light led-based visible light communication", The 4th International Conference on Systems and Informatics (ICSAI), Hangzhou, pp.1035-1039, 2017. https://doi.org/10.1109/ICSAI.2017.8248438.

[8] Y.Q. Wang, Y.G. Wang, N. Chi, J.J. Yu, and H.L. Shang, "Demonstration of $575 \mathrm{M} \mathrm{b} / \mathrm{s}$ downlink and $225 \mathrm{Mb} / \mathrm{s}$ uplink bi-directional SCM-WDM visible light communication using RGB LED and phosphor-based LED", Optics Express, vol. 21, no. 1, pp.1203-1208, 2013.

https://doi.org/10.1364/OE.21.001203. 
[9] F.M. Wu, C.T. Lin, C.C. Wei, C.W. Chen, Z.Y. Chen, and H.T. Huang, "3.22Gb/s WDM visible light communication of a single RGB LED employing carrier-less amplitude and phase modulation", Optical Fiber Communication Conference / National Fiber Optic Engineers Conference, OSA Technical Digest, Optical Society of America, paper OTh1G.4, 2013. https://doi.org/10.1364/OFC.2013.OTh1G.4.

[10] YQ. Wang, X.X. Huang, J.W. Zhang, Y.G. Wang, and N. Chi, "Enhanced performance of visible light communication employing 512 QAM NSC-FDE and DD-LMS", Optics Express, vol. 22, no. 13, pp.15328-15334, 2014.

https://doi.org/10.1364/oe.22.015328.

[11] M. Uysal and H. Nouri, "Optical wireless communications - An emerging technology", The 16th International Conference on Transplant Optical Networks (ICTON), Graz, pp.1-7, 2014, https://doi.org/10.1109/ICTON.2014.6876267.

[12] A. Pradana, N. Ahmadi and T. Adiono, "Design and implementation of visible light communication system using pulse width modulation", The 5th International Conference on Electrical Engineering and Informatics (ICEEI), Denpasar, pp.25-30, 2015. https://doi.org/10.1109/ICEEI.2015.7352464.

[13] J. Vučič, C. Kottke, S. Nerreter, K. Langer, and J.W. Walewski, " $513 \mathrm{mbit} / \mathrm{s}$ visible light communications link based on DMT-modulation of a white led", Journal of Lightwave Technology, vol. 28, no. 24, pp.3512-3518, 2010.

https://doi.org/10.1109/JLT.2010.2089602.

[14] A.E. Ibhaze, F.O. Edeko, and P.E. Orukpe, "A Signal amplification-based transceiver for visible light communication", Journal of Engineering, vol. 26, no. 11, 2020. https://doi.org/10.31026/j.eng.2020.11.08.

[15] X. Li, N. Bamiedakis, X. Guo, J.J.D. McKendry, E. Xie, R. Ferreira, E. Gu, MD. Dawson, R.V. Penty, and I.H. White, "Wireless visible light communications employing feed-forward preequalization and PAM-4 modulation", Journal of Lightwave Technology, vol. 34, no. 8, pp.2049-2055, 2016. https://doi.org/10.1109/JLT.2016.2520503.

[16] N. Fujimoto and H. Mochizuki, "477Mbit/s visible light transmission based on OOK-NRZ modulation using a single commercially available visible LED and a practical LED driver with a pre-emphasis circuit", Optical Fiber Communication Conference / National Fiber Optic Engineers Conference (OFC/NFOEC), Anaheim, CA, paper JTh2A.73, 2013.

https://doi.org/10.1364/NFOEC.2013.JTh2A.73.
[17] C.W. Hsu, Y. Hsu, G.H. Chen, L.Y. Wei, C.W. Chow, I.C. Lu, Y.L. Liu, and C.H. Yeh, "Mitigation of LED nonlinearity using adaptive equalization for visible light communications", Asia Communications and Photonics Conference (ACP), paper Su2A.52, 2017. https://doi.org/10.1364/ACPC.2017.Su2A.52.

[18] T. Komine, and M. Nakagawa, "Fundamental analysis for visible-light communication system using LED lights", IEEE Transactions on Consumer Electronics, vol. 50, no. 1, pp.100-107, 2004. https://doi.org/10.1109/TCE.2004.1277847.

[19] P. Amirshahi, and M. Kavehrad, "Broadband access over medium and low voltage power-lines and use of white light-emitting diodes for indoor communications", The 3rd IEEE Consumer Communications and Networking Conference, Las Vegas, NV, USA, pp.897-901, 2006. https://doi.org/10.1109/CCNC.2006.1593168.

[20] K. Lee and H. Park, "Modulations for visible light communications with dimming control", IEEE Photonics Technology Letters, vol. 23, no. 16, pp.1136-1138, 2011.

https://doi.org/10.1109/LPT.2011.2157676.

[21] L.T. Dung, S. Jo, and B. An, "Demonstration of lowcomplexity LED-to-LED two-way visible light communication system", International Symposium on Computer, Consumer and Control (IS3C), Xi'an, pp.216-219, 2016.

https://doi.org/10.1109/IS3C.2016.65.

[22] D. O'Brien, H.L. Minh, L. Zeng, G. Faulkner, K. Lee, D. Jung, Y. Oh, and E.T. Won, "Indoor visible light communications: challenges and prospects", Free-Space Laser Communications VIII (FSLC), Proceeding SPIE vol. 7091, San Diego, California, 2008. https://doi.org/10.1117/12.799503.

[23] Y. He, L. Ding, Y. Gong, and Y. Wang, "Real-time audio and video transmission system based on visible light communication", Optics and Photonics Journal, vol. 3, no. 2B, pp.153-157, 2013. https://doi.org/10.4236/opj.2013.32B037.

[24] Y. Zhao, and J. Vongkulbhisal, "Design of visible light communication receiver for on-off keying modulation by adaptive minimum-voltage cancellation", Engineering Journal, vol. 17, no. 4, pp. 126-129, 2013. http://dx.doi.org/10.4186/ej.2013.17.4.125. 